\title{
Longitudinal strain assessed by cardiac magnetic resonance correlates to hemodynamic findings in patients with severe aortic stenosis and predicts positive remodeling after transcatheter aortic valve replacement
}

\author{
Dominik Buckert $^{1}{ }^{10} \cdot$ Maciej Cieslik $^{1} \cdot$ Raid Tibi $^{1} \cdot$ Michael Radermacher $^{1}$. \\ Volker Rasche $^{1}$ - Peter Bernhardt ${ }^{1}$ • Vinzenz Hombach ${ }^{1}$ - Wolfgang Rottbauer ${ }^{1}$. \\ Jochen Wöhrle ${ }^{1}$
}

Received: 3 May 2017 / Accepted: 10 August 2017 / Published online: 14 August 2017

(C) The Author(s) 2017. This article is an open access publication

\begin{abstract}
Aims To assess left-ventricular strain parameters before and after transcatheter aortic valve replacement (TAVR) by feature tracking cardiac magnetic resonance imaging (FT $\mathrm{CMR}$ ) and to correlate the findings to hemodynamic state and left-ventricular remodeling.

Methods and results Patients with symptomatic AS underwent FT CMR before and after TAVR. Patients were carefully evaluated by a comprehensive work-up including CMR, echocardiography and left and right heart catheterization. Thirty patients formed the study population. Highflow/high-gradient $(\mathrm{HF} / \mathrm{HG})$ aortic stenosis was diagnosed in 11 patients (36.7\%), 6 patients (20.0\%) exhibited lowflow/low-gradient AS (LF/LG) and 13 patients (43.3\%) were classified to have so-called paradoxical low-flow/ low-gradient (PLF/LG) AS. The HF/HG patients had a significantly reduced longitudinal strain which recovered after TAVR $(-12.67 \pm 4.60$ to $-15.46 \pm 5.61 \%, p=0.048)$. In the LF/LG group, an even more pronounced reduction of longitudinal strain and also an impairment of longitudinal velocity could be observed. Both parameters improved after therapy (strain: $-5.06 \pm 4.25$ to $-8.02 \pm 3.28 \%, p=0.045$; velocity: $25.33 \pm 9.63$ to $37.13 \pm 11.64 \mathrm{~mm} / \mathrm{s}, p=0.042$ ). Patients with PLF/LG showed preserved longitudinal strain but a reduction of longitudinal velocity similar to the $\mathrm{LF} / \mathrm{LG}$ group. These patients did not show a significant improvement of strain parameters after TAVR. Longitudinal velocity exhibited the highest predictive power for the identification of a low-flow state (sensitivity $75 \%$, specificity $80 \%$ ).
\end{abstract}

Dominik Buckert

dominik.buckert@uniklinik-ulm.de

1 Department of Internal Medicine II, University of Ulm, Albert-Einstein-Allee 23, 89081 Ulm, Germany
Conclusion Improvement of longitudinal strain parameters after TAVR is dependent on the initial hemodynamically defined AS subgroup.

Keywords Cardiac magnetic resonance imaging - Strain imaging $\cdot$ Cardiac mechanics $\cdot$ Severe aortic stenosis · Transcatheter aortic valve replacement

\section{Introduction}

Aortic stenosis (AS) is one of the most common valvular pathologies in western populations, especially in the elderly [1]. Once severe AS causes symptoms, indication for aortic valve replacement is given [2]. In patients at high surgical risk, transcatheter aortic valve replacement (TAVR) has been shown to be superior to surgical valve replacement [3-6].

The current definition of severe AS comprises either an aortic valve area (AVA) $\leq 1.0 \mathrm{~cm}^{2}$, or a mean pressure gradient (MPG) $\geq 40 \mathrm{mmHg}$ or a peak velocity $\geq 4 \mathrm{~m} / \mathrm{s}[2,7]$. Since MPG and peak velocity are highly dependent on flow across the valve, premise of this definition is a hemodynamic situation in which a normal flow and usually a normal left-ventricular ejection fraction (LVEF) are given [8]. Patients exhibiting these features are called to have 'highflow/high-gradient AS (HF/HG)' [7, 8]. However, there are specific subsets of patients suffering from severe AS in which the hemodynamic situations are different. In patients with reduced LVEF, either caused by the AS itself or other cardiac pathologies, a 'low-flow/low-gradient AS (LF/LG)' may be present due to decreased stroke volume and low flow across the valve [9]. There is a third important subset of patients in which the chronic pressure overload caused by longstanding AS leads to a progressive concentric hypertrophy and a severe diastolic dysfunction [10]. These patients 
also show decreased stroke volumes and flow despite LVEF is preserved and, thus, have a 'paradoxical low-flow/lowgradient AS (PLF/LG)' [11]. Correct diagnosis and identification of the specific AS pathology are of great importance, since outcomes and prognosis of the particular subpopulations and their response to aortic valve replacement differ [12].

Strain imaging is an emerging technique for the noninvasive evaluation of global and regional left ventricular function [13]. Its ability to characterize contractility patterns in different hemodynamic situations has been proven, especially for longitudinal strain [14]. Accordingly, it was successfully used to assess functional properties of different AS pathologies as well as the specific changes after valve replacement [15]. In most of the studies forming the evidence base, strain values were derived from 2D speckletracking echocardiography. Though this imaging technique is highly accepted and widely available, it exhibits several limitations such as relative low inter-reader and intra-reader reproducibility and the need for an appropriate 'acoustic window' $[16,17]$.

With its high spatial resolution, good image contrast, lack of ionizing radiation and favorable reproducibility, cardiac magnetic resonance imaging (CMR) plays an increasing role in the diagnostic management of patients suffering from valve pathologies $[18,19]$. It is considered the gold standard for the evaluation and quantification of left ventricular functional parameters such as volumes and LVEF [20-22]. Recently, feature tracking (FT) techniques have been introduced to derive strain parameters from conventional CMR cine images [23, 24].

The objective of our study was to assess left ventricular longitudinal strain parameters by FT CMR before and after TAVR and to correlate the findings to the underlying AS pathology.

\section{Methods}

\section{Study populations}

Patients with symptomatic severe AS undergoing TAVR were enrolled from 2014 to 2015 [25]. All patients were considered eligible unless they exhibited predefined exclusion criteria such as cardiac or respiratory instability, metal implants or devices unsuitable for CMR, concomitant limiting disease, allergy against gadolinium-based contrast agents or severely impaired renal function (estimated glomerular filtration rate $<30 \mathrm{ml} / \mathrm{min}$ ). An age- and sex-matched healthy control population was derived from a database formed within another project.

All TAVR patients were carefully evaluated concerning other cardiac diseases such as coronary heart disease, relevant other valvular heart disease, hypertrophic or dilative cardiomyopathy, diastolic dysfunction and inflammatory heart diseases. For the healthy controls, these conditions were rigorously ruled out. The study was approved by the institutional ethics committee (clinicaltrials.gov: NCT02162069). Written informed consent was obtained from every patient as well as from the healthy controls.

\section{Hemodynamic evaluation}

All patients received a comprehensive diagnostic work-up prior to TAVR including CMR, transthoracic echocardiography and hemodynamic left and right heart catheterization. Pressure gradients across the aortic valve were invasively assessed by simultaneous measurements in the left ventricle and aorta. Hemodynamic evaluation included the invasive and non-invasive measurement of pressure gradients, pulmonary artery pressures, systemic pressures, stroke volumes, cardiac output and cardiac power index [26]. A 'low-flow state' was defined as a left-ventricular stroke index (LVSVi) $\leq 35 \mathrm{ml} / \mathrm{m}^{2}$ and/or a cardiac index (Ci) $\leq 3.0 \mathrm{l} / \mathrm{min}$ [27]. These parameters can be assessed by invasive measurement as well as non-invasively by CMR and echo. Every patient was carefully evaluated taking into account the results of every modality as well as supporting features (left-ventricular volumes, diastolic dysfunction) in case of conflicting results. Plausibility of the results of each modality was rated individually for each patient. In conclusion of all findings, hemodynamic state of each patient was defined according to the current guidelines and recommendations $(\mathrm{HF} / \mathrm{HG}, \mathrm{LF} /$ LG, PLF/LG) [26-28]. Assignment to a specific AS subgroup was done before strain assessment was performed in order to avoid bias.

\section{CMR examination}

All patients received CMR examinations within 5 days before and at 3 months after TAVR. CMR imaging was performed on a 1.5-T whole body clinical magnetic resonance scanner (Achieva 1.5T, Philips Medical Systems, Best, Netherlands) using a 32-channel phased-array receiver coil. CMR examinations were carried out in concordance with current guidelines [29, 30]. A steady-state free precession sequence (SSFP, repetition time $3.4 \mathrm{~ms}$, echo time $1.7 \mathrm{~ms}$, voxel size $1.6 \times 1.6 \mathrm{~mm}$, flip-angle $\alpha 55^{\circ}$, slice thickness $8 \mathrm{~mm}$, acquisition in end-expiratory breath-hold, 32 cardiac phases) was used for functional imaging of the left and right ventricle in long- and short-axis orientation.

\section{CMR analysis}

Two experienced readers, blinded to patient history and hemodynamic findings, performed offline image analysis. 
Epi- and endocardial contours were drawn manually in the long- and short-axis-oriented SSFP-images. Basic functional and strain parameters (strain, systolic strain rate, displacement, systolic velocity) were derived from the SSFP cine images using the dedicated software $\mathrm{cvi}^{42 \circledast}$ (Version 5.2, Circle Cardiovascular Imaging, Calgary, Canada). Strain parameters were assessed globally in longitudinal, radial and circumferential orientation for the left ventricle according to the current recommendations [31, 32]. A healthy control population was set up for the validation of the used approach and the yielded results.

\section{Statistical analysis}

To test the correlation between two categorical classification factors, the Chi squared test was applied. Continuous variables were tested for normal distribution by the D'Agostino-Pearson test. Variables with normal distribution were reported as mean \pm standard deviation and a two-tailed $t$ test (either for paired or independent samples) was used for comparison. Variables without normal distribution were reported as median with percentiles and compared by the Mann-Whitney $U$ rank sum test. Intra-class correlation coefficient and inter-rater agreement were assessed to evaluate reproducibility and inter-rater reliability of strain imaging analyzes. To determine the accuracy of the strain-based lowflow state classification, a receiver operating characteristic (ROC) curve analysis was performed. A $p$ value $<0.05$ was considered significant. Statistical analyses were performed using commercially available software (Stata 13, College Station, USA, MedCalc, Mariakerke, Belgium).

\section{Results}

\section{Study populations}

Thirty patients suffering from severe AS were enrolled in this study. Mean age was $78.8 \pm 5.9$ years, $50.0 \%$ were men $(n=15)$. Mean NYHA class before TAVR was $3.1 \pm 0.5$. The score of the Society of Thoracic Surgeons (STS) for mortality was $4.7 \pm 3.0 \%$ [33]. The EURO II score was $5.4 \pm 3.8 \%$ [34]. Clinical baseline characteristics are depicted in Table 1. The control group consisted of 40 individuals ( 20 men and 20 women) at a mean age of $74.4 \pm 2.8$ years. None of the patients had paraprosthetic aortic regurgitation more than 'trace' or required permanent pacemaker stimulation after TAVR.

\section{Hemodynamic evaluation}

An HF/HG AS was observed in 11 patients (36.7\%). Six subjects $(20.0 \%)$ were categorized in the LF/LG group.
Table 1 Baseline characteristics

\begin{tabular}{lll}
\hline & \multicolumn{2}{l}{ Total cohort $(n=30)$} \\
\hline Age (years) & $78.8 \pm 5.9$ & Mean \pm SD \\
Sex $($ men $)$ & $15(50.0)$ & $\mathrm{N},(\%)$ \\
BMI $\left(\mathrm{kg} / \mathrm{m}^{2}\right)$ & $27.4 \pm 5.1$ & Mean $\pm \mathrm{SD}$ \\
Hypertension & $29(96.7)$ & $\mathrm{N},(\%)$ \\
Hyperlipoproteinaemia & $24(80.0)$ & $\mathrm{N},(\%)$ \\
Diabetes mellitus & $11(36.7)$ & $\mathrm{N},(\%)$ \\
Coronary artery disease & $24(80.0)$ & $\mathrm{N},(\%)$ \\
Atrial fibrillation & $13(43.3)$ & $\mathrm{N},(\%)$ \\
NYHA class & $3.1 \pm 0.6$ & Mean $\pm \mathrm{SD}$ \\
EURO score II & $5.4 \pm 3.8$ & Mean $\pm \mathrm{SD}$ \\
STS-Score & $4.7 \pm 3.0$ & Mean \pm SD \\
\hline
\end{tabular}

BMI body mass index, NYHA New York Heart Association, STS Society of Thoracic Surgeons

A PLF/LG situation was diagnosed in 13 cases (43.3\%). In the $\mathrm{HF} / \mathrm{HG}$ group, MPG was $47.5 \pm 16.1 \mathrm{mmHg}$, LVSVi was $45.3 \pm 6.4 \mathrm{ml} / \mathrm{m}^{2}$, Ci was $3.3 \pm 0.58 \mathrm{l} / \mathrm{min}$ and AVA was $0.56 \pm 0.13 \mathrm{~cm}^{2}$. Patients in the LF/LG group showed a severely reduced LVEF with $26.3 \pm 7.2 \%$, an MPG of $28.8 \pm 9.5 \mathrm{mmHg}$, an LVSVi of $30.4 \pm 4.9 \mathrm{ml} / \mathrm{m}^{2}$, a Ci of $2.5 \pm 0.71 \mathrm{l} / \mathrm{min}$ and an AVA of $0.80 \pm 0.14 \mathrm{~cm}^{2}$. In the PLF/LG group, a preserved LVEF could be observed with $63.7 \pm 8.4 \%$. These patients exhibited an MPG of $26.1 \pm 5.7 \mathrm{mmHg}$, an LVSVi of $32.7 \pm 5.9 \mathrm{ml} / \mathrm{m}^{2}$, a Ci of $2.7 \pm 0.47 \mathrm{l} / \mathrm{min}$ and an AVA of $0.77 \pm 0.22 \mathrm{~cm}^{2}$. In the PLF/LG group, 7 patients exhibited only mild hypertrophy $(\leq 0.8 \mathrm{~g} / \mathrm{ml})$ as defined by the left-ventricular volume/mass ratio. Five of them (71.4\%) were women. Table 2 shows the hemodynamic characteristics of the total cohort and the different AS entities.

\section{Presence of late gadolinum enhancement, coronary artery disease and concomitant valve pathologies}

Late gadolinium enhancement was assessed in the initial CMR study. Fourteen patients ( $46.7 \%$ of the total study group) were LGE positive. Eleven of them showed LGE patterns consistent with fibrosis (36.7\%). Though there was no statistically significant difference concerning distribution of LGE throughout the AS subgroups, patients predominantly tended to be LGE positive in the HF/HG and LF/LG group (HF/HG: $n=5,45.5 \%$; LF/LG: $n=4,66.7 \%$; PLF/LG: $n=2,15.4 \%$; $p=0.07$ for distribution). A relevant coronary artery disease was present in $n=24(80 \%)$ of the total study cohort with no significant difference concerning the distribution between the subgroups (HF/HG: $n=8,72.7 \%$; LF/LG: $n=5,83.3 \%$; PLF/LG: $n=11,84.6 \% ; p=0.47)$. In Table 3, frequencies of late gadolinium enhancement and coronary artery disease are depicted. None of the patients had a concomitant aortic or mitral valve regurgitation higher than grade 1 . 
Table 2 Hemodynamic characteristics

\begin{tabular}{ccccc}
\hline & Total cohort & HF/HG & LF/LG & PLF/LG \\
\hline LVEF (\%) & $56.7 \pm 18.4$ & $65.0 \pm 13.2$ & $26.3 \pm 7.2$ & $63.7 \pm 8.4$ \\
$\begin{array}{c}\text { LVEDVi } \\
\left(\mathrm{ml} / \mathrm{m}^{2}\right)\end{array}$ & $79.5 \pm 30.9$ & $73.0 \pm 21.3$ & $123.3 \pm 36.1$ & $64.8 \pm 12.43$ \\
$\begin{array}{c}\mathrm{LVSVi} \\
\left(\mathrm{ml} / \mathrm{m}^{2}\right)\end{array}$ & $40.0 \pm 8.3$ & $45.3 \pm 6.4$ & $30.4 \pm 4.9$ & $32.72 \pm 5.9$ \\
$\mathrm{Ci}$ & $2.9 \pm 0.66$ & $3.3 \pm 0.58$ & $2.5 \pm 0.71$ & $2.7 \pm 0.47$ \\
$\mathrm{RVEF}(\%)$ & $60.4 \pm 12.2$ & $67.8 \pm 8.1$ & $46.8 \pm 13.0$ & $60.3 \pm 9.7$ \\
$\mathrm{RVEDVi}$ & $68.7 \pm 15.4$ & $62.6 \pm 11.0$ & $80.5 \pm 22.6$ & $68.3 \pm 12.5$ \\
$\left(\mathrm{ml} / \mathrm{m}^{2}\right)$ & & & & \\
$\mathrm{MPG}$ & $34.0 \pm 14.6$ & $47.5 \pm 16.1$ & $28.8 \pm 9.5$ & $26.1 \pm 5.7$ \\
$(\mathrm{mmHg})$ & & & & \\
$\mathrm{AVA}\left(\mathrm{cm}{ }^{2}\right)$ & $0.69 \pm 0.21$ & $0.56 \pm 0.13$ & $0.80 \pm 0.14$ & $0.77 \pm 0.22$ \\
$\begin{array}{c}\mathrm{LVEDP} \\
(\mathrm{mmHg})\end{array}$ & $20.7 \pm 6.2$ & $19.7 \pm 6.7$ & $26.5 \pm 2.1$ & $20.3 \pm 6.1$ \\
\hline
\end{tabular}

Values are mean \pm standard deviation

$A V A$ aortic valve area, $C I$ cardiac index, $H F / H G$ high-flow/high-gradient aortic stenosis, $L F / L G$ low-flow/low-gradient aortic stenosis, $L V E D V i$ left-ventricular end-diastolic volume index, $L V E D P$ leftventricular end-diastolic pressure, $L V E F$ left ventricular ejection fraction, $L V S V i$ left-ventricular stroke volume index, $M P G$ mean pressure gradient, $P L F / L G$ paradoxical low-flow/low-gradient aortic stenosis, $R V E D V I$ right-ventricular end-diastolic volume index, $R V E F$ rightventricular ejection fraction

\section{Strain imaging before TAVR}

Longitudinal strain parameters were assessed with excellent reproducibility and reliability as detailed in Table 4.

Table 5 shows the results of strain analysis for the total cohort, the AS subgroups and the healthy controls. The HF/ HG patients showed a slightly but statistically significant reduced global longitudinal strain in comparison to the controls $(-12.67 \pm 4.60$ vs. $-15.91 \pm 1.96 \%, p=0.001)$. Displacement and velocity were not significantly different. In comparison to the controls and the HF/HG group, the subjects of the LF/LG group showed not only a significant reduction of strain (comparison to HF/HG: $-5.06 \pm 4.25$ vs. $-12.67 \pm 4.60 \%, p=0.005$; comparison to controls: $-5.06 \pm 4.25$ vs. $-15.91 \pm 1.96 \%, p<0.0001)$ but also of longitudinal velocity (comparison to HF/HG: $25.33 \pm 9.63$ vs. $47.24 \pm 16.63 \mathrm{~mm} / \mathrm{s}, p=0.01$; comparison to controls: $25.33 \pm 9.63 \mathrm{~mm} / \mathrm{s}$ vs. $42.02 \pm 12.39, p=0.003)$. The PLF/ LG group showed preserved strain (comparison to controls: $-15.80 \pm 4.56$ vs. $-15.91 \pm 1.96 \%, p=0.90$ ) but a reduction in velocity comparable to the LF/LG group (comparison to controls: $29.76 \pm 9.98 \mathrm{~mm} / \mathrm{s}$ vs. $42.02 \pm 12.39$, $p=0.002$ ). Mean strain and velocity values for the subgroups and controls are depicted in Fig. 1.
Table 3 Presence of coronary artery disease and late gadolinium enhancement

\begin{tabular}{lllll}
\hline & Total cohort & HF/HG & LF/LG & PLF/LG \\
\hline Presence of Coronary artery disease $(n, \%)$ & $24(80.0)$ & $8(72.7)$ & $5(83.3)$ & $11(84.6)$ \\
Late gadolinium enhancement $(n, \%)$ & $14(46.7)$ & $7(54.5)$ & $6(100)$ & $2(15.4)$ \\
Consistent with fibrosis & $11(36.7)$ & $5(45.5)$ & $4(66.7)$ & $2(15.4)$ \\
\hline
\end{tabular}

$H F / H G$ high-flow/high-gradient aortic stenosis, $L F / L G$ low-flow/low-gradient aortic stenosis, $P L F / L G$ paradoxical low-flow/low-gradient aortic stenosis

\begin{tabular}{|c|c|c|}
\hline & Intra-class correlation & Inter-reader agreement \\
\hline \multicolumn{3}{|l|}{ Longitudinal } \\
\hline Peak strain $(\%)$ & $\begin{array}{l}0.94 \\
\text { CI }[0.70 ; 0.98]\end{array}$ & $\begin{array}{l}\text { Kappa: } 0.94 ; \\
\text { Standard error: } 0.02 \\
\text { CI }[0.90 ; 0.97]\end{array}$ \\
\hline Peak strain rate systolic $(\% / \mathrm{s})$ & $\begin{array}{l}0.90 \\
\text { CI }[0.73 ; 0.95]\end{array}$ & $\begin{array}{l}\text { Kappa: } 0.83 \\
\text { Standard error: } 0.05 \\
\text { CI }[0.73 ; 0.93]\end{array}$ \\
\hline Peak displacement (mm) & $\begin{array}{l}0.98 \\
\text { CI [0.96; 0.99] }\end{array}$ & $\begin{array}{l}\text { Kappa: } 0.98 \\
\text { Standard error: } 0.01 \\
\text { CI }[0.96 ; 0.99]\end{array}$ \\
\hline Peak velocity $(\mathrm{mm} / \mathrm{s})$ & $\begin{array}{l}0.98 \\
\text { CI [0.95; 0.99] }\end{array}$ & $\begin{array}{l}\text { Kappa: } 0.95 \\
\text { Standard error: } 0.03 \\
\text { CI }[0.90 ; 1.00]\end{array}$ \\
\hline
\end{tabular}

CI confidence interval
Table 4 Intraclass correlation and inter-reader agreement of longitudinal strain 
Table 5 Strain imaging before transcatheter aortic valve replacement

\begin{tabular}{|c|c|c|c|c|c|}
\hline & Total cohort & $\mathrm{HF} / \mathrm{HG}$ & LG/LG & PLF/LG & Controls \\
\hline \multicolumn{6}{|l|}{ Longitudinal } \\
\hline Peak strain $(\%)$ & $-12.61 \pm 5.58$ & $-12.67 \pm 4.60$ & $-5.06 \pm 4.25$ & $-15.80 \pm 4.56$ & $-15.91 \pm 1.96$ \\
\hline Peak strain rate systolic $(\% / s)$ & $79.20 \pm 37.39$ & $97.79 \pm 47.93$ & $42.69 \pm 14.53$ & $81 \pm 28.48$ & $91.38 \pm 23.54$ \\
\hline Peak displacement (mm) & $3.62 \pm 1.42$ & $3.61 \pm 1.21$ & $2.40 \pm 1.91$ & $3.98 \pm 1.21$ & $3.30 \pm 1.28$ \\
\hline Peak velocity $(\mathrm{mm} / \mathrm{s})$ & $35.30 \pm 16.01$ & $47.24 \pm 16.63$ & $25.33 \pm 9.63$ & $29.76 \pm 9.98$ & $42.02 \pm 12.39$ \\
\hline \multicolumn{6}{|l|}{ Radial } \\
\hline Peak strain $(\%)$ & $29.20 \pm 13.50$ & $\begin{array}{c}30.94 \pm \\
10.09\end{array}$ & $12.05 \pm 7.37$ & $35.65 \pm 11.76$ & $47.16 \pm 11.37$ \\
\hline Peak strain rate systolic $(\% / \mathrm{s})$ & $221.46 \pm 95.62$ & $\begin{array}{c}248.46 \pm \\
78.26\end{array}$ & $91.69 \pm 64.79$ & $258.51 \pm 68.33$ & $291.15 \pm 93.07$ \\
\hline Peak displacement (mm) & $4.03 \pm 2.03$ & $4.00 \pm 2.17$ & $2.07 \pm 1.31$ & $4.96 \pm 1.56$ & $6.84 \pm 0.87$ \\
\hline Peak velocity $(\mathrm{mm} / \mathrm{s})$ & $26.46 \pm 10.79$ & $29.95 \pm 10.98$ & $12.53 \pm 6.10$ & $29.95 \pm 6.65$ & $40.77 \pm 8.61$ \\
\hline \multicolumn{6}{|l|}{ Circumferential } \\
\hline Peak strain (\%) & $-10.37 \pm 9.02$ & $-9.13 \pm 11.78$ & $-3.00 \pm 4.47$ & $-14.83 \pm 4.75$ & $-18.46 \pm 2.41$ \\
\hline Peak strain rate systolic $(\% / s)$ & $-93.02 \pm 48.78$ & $-97.36 \pm 48.24$ & $-27.00 \pm 18.24$ & $-119.84 \pm 26.38$ & $-105.13 \pm 25.40$ \\
\hline Peak displacement (deg) & $0.04 \pm 1.79$ & $-0.36 \pm 2.06$ & $0.73 \pm 1.61$ & $0.03 \pm 1.70$ & $-0.11 \pm 0.15$ \\
\hline Peak velocity (deg/s) & $-4.49 \pm 21.91$ & $-4.91 \pm 23.80$ & $6.22 \pm 21.84$ & $-9.08 \pm 20.23$ & $-1.29 \pm 0.95$ \\
\hline
\end{tabular}

Values are mean \pm standard deviation

$H F / H G$ high-flow/high-gradient aortic stenosis, $L F / L G$ low-flow/low-gradient aortic stenosis, $P L F / L G$ paradoxical low-flow/low-gradient aortic stenosis

Fig. 1 Global longitudinal strain and global longitudinal velocity in dependency of hemodynamic subgroups. In comparison to the $\mathrm{HF} / \mathrm{HG}$ group, a significant reduction of strain and velocity could be observed for the LF/LG group. Patients in the PLF/LG group showed strain comparable to the $\mathrm{HF} / \mathrm{HG}$ group but a reduction in velocity similar to the $\mathrm{LF} / \mathrm{LG}$ group $(H F / H G$ high-flow/highgradient aortic stenosis, $L F / L G$ low-flow/low-gradient aortic stenosis, $P L F / L G$ paradoxical low-flow/low-gradient aortic stenosis)

\section{Mean values of longitudinal strain and velocity in dependency of hemodynamically situation}

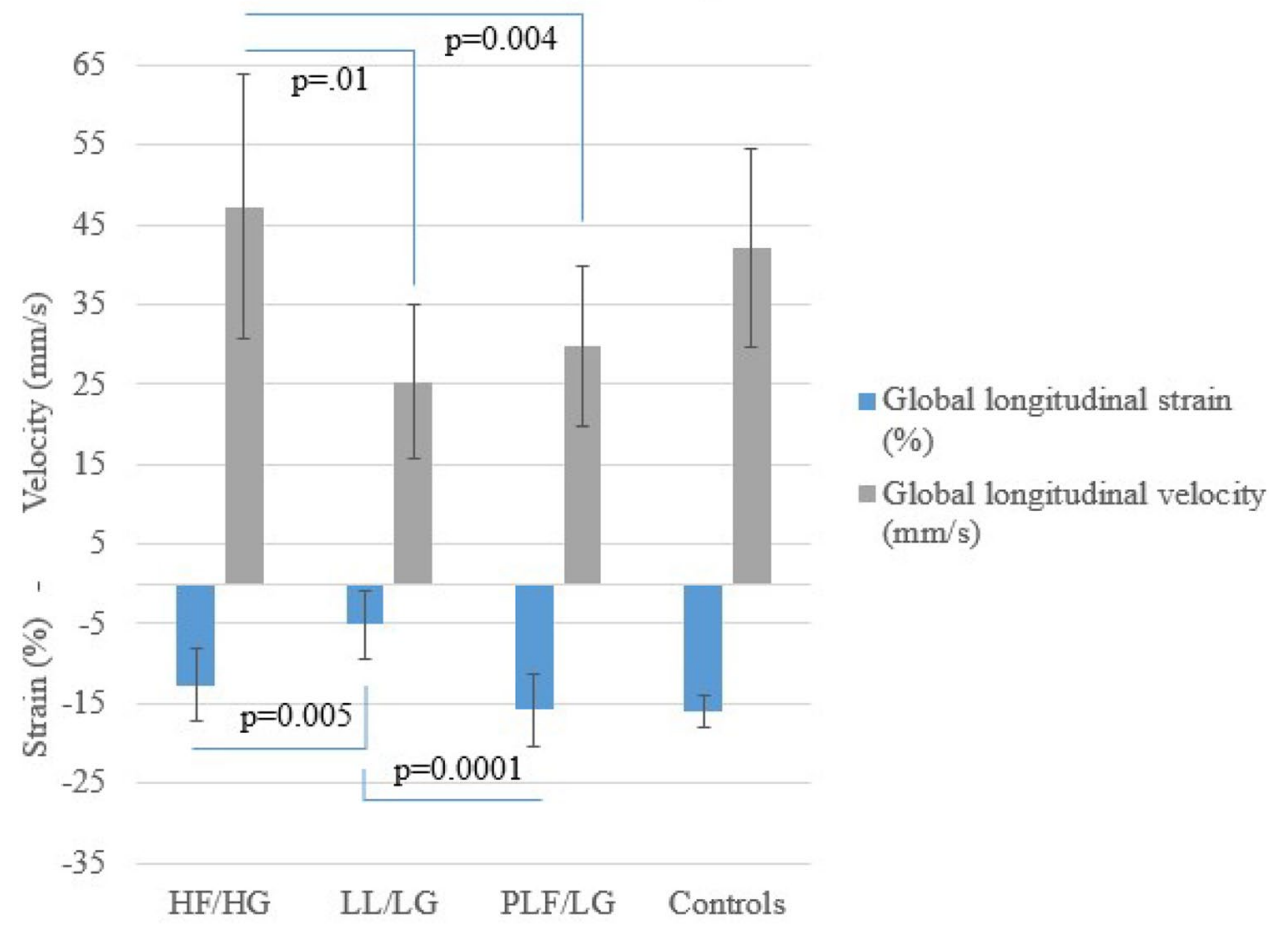


Deformation parameters in radial and circumferential orientation were significantly reduced for all AS subgroups in comparison to the healthy control group (Table 5). A correlation between radial and circumferential strain and hemodynamic state could not be observed.

To assess the predictive power of global longitudinal velocity for the identification of a 'low-flow' state, an ROC curve analysis was performed. The result is depicted in

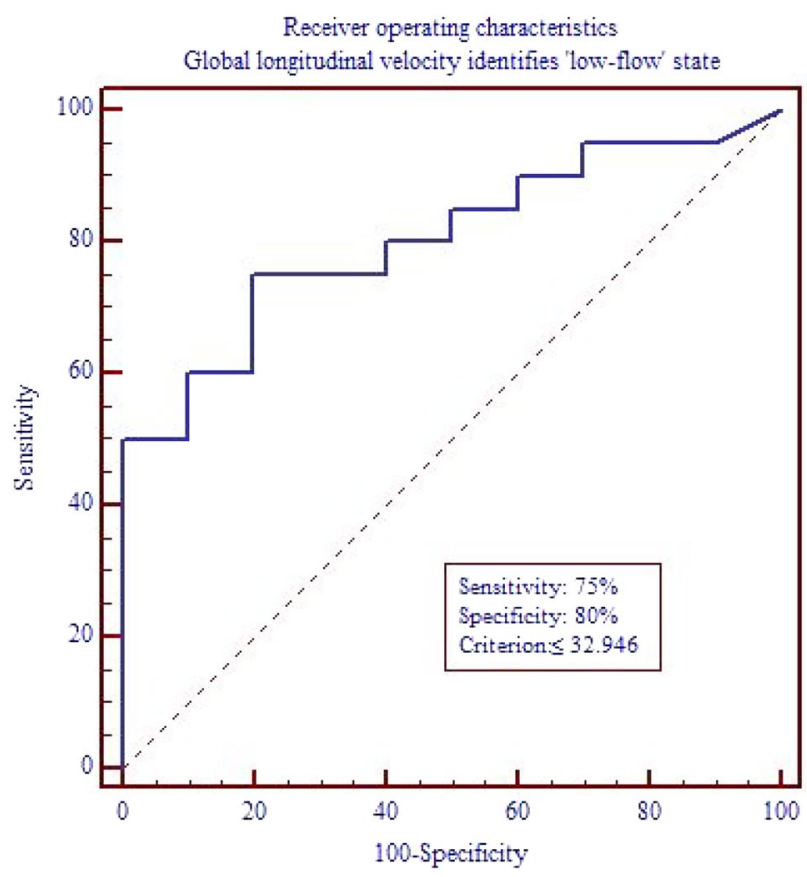

Fig. 2 Receiver operating characteristics analysis. By the use of a cutoff criterion of $\leq 32.946 \mathrm{~mm} / \mathrm{s}$, a 'low-flow' state could be predicted by global longitudinal velocity with a sensitivity of $75 \%$ and a specificity of $80 \%$
Fig. 2. By the use of a cutoff criterion of $\leq 32.946 \mathrm{~mm} / \mathrm{s}$, global longitudinal velocity correctly predicted a 'low-flow' situation with a sensitivity of $75 \%$ and a specificity of $80 \%$.

\section{Changes after TAVR}

Tables 6 and 7 show changes in selected clinical variables (Table 6) and strain parameters (Table 7) in dependency of the initial AS subgroup. For the total study cohort, NTproBNP before and after TAVR was $3804.1 \pm 3909.4$ and $3339.4 \pm 3533.6 \mathrm{pg} / \mathrm{ml}(p=0.36)$, respectively. Though statistical significance was not reached, NT-proBNP levels were lower after TAVR for the HF/HG and LF/LG groups (HF/HG: $3823.9 \pm 3966.3$ vs. $2669.7 \pm 2163.26, p=0.28$; LF/LG: $6822.5 \pm 4844.9$ vs. $6580.3 \pm 6146.5 \mathrm{pg} / \mathrm{ml}$, $p=0.85)$. In the PLF/LG group, NT-proBNP levels were higher after the intervention (PLF/LG: $2056.6 \pm 2474.7$ vs. $2253.0 \pm 2033.0 \mathrm{pg} / \mathrm{ml}, p=0.65)$.

Patients in the HF/HG group additionally showed a significant increase in global longitudinal strain $(-12.67 \pm 4.60$ to $-15.46 \pm 5.61 \%, p=0.048)$ and longitudinal displacement $(3.44 \pm 1.17$ to $5.09 \pm 1.46 \mathrm{~mm}$, $p=0.016)$. These patients thus reached values comparable to the healthy controls (comparison of longitudinal strain after TAVR to controls: $-15.46 \pm 6.61$ vs. $-15.91 \pm 1.96 \%, p=0.67)$. A significant increase in longitudinal strain could also be observed for the LF/LG group $(-5.06 \pm 4.25$ to $-8.02 \pm 3.28 \%, p=0.045)$. These patients also exhibited a significant improvement in longitudinal velocity $(25.33 \pm 9.63 \mathrm{~mm} / \mathrm{s}$ to $37.13 \pm 11.64$, $p=0.042)$ and LVEF $(26.3 \pm 7.23$ to $35.5 \pm 13.69$, $p=0.027)$. There was no improvement in strain parameters for patients of the PLF/LG group.

Table 6 Changes after transcatheter aortic valve replacement in dependency of hemodynamic situation

\begin{tabular}{lccc}
\hline & Before TAVR & After TAVR & Change (paired differences) \\
\hline High-flow/high-gradient aortic stenosis & & & \\
NYHA class & $3.4 \pm 0.55$ & $2.0 \pm 0.71$ & $-1.4 \pm 0.89$ \\
Left-ventricular ejection fraction (\%) & $66.8 \pm 12.41$ & $69.3 \pm 8.77$ & $2.5 \pm 13.47$ \\
NT-proBNP (pg/ml) & $3823.9 \pm 3966.3$ & $2669.7 \pm 2163.26$ & $1154.2 \pm 2769.6$ \\
Low-flow/low-gradient aortic stenosis & & & 0.025 \\
NYHA class & $3.31 \pm 0.48$ & $2.08 \pm 0.64$ & $-1.23 \pm 0.83$ \\
Left-ventricular ejection fraction (\%) & $26.3 \pm 7.23$ & $35.5 \pm 13.69$ & $9.17 \pm 7.22$ \\
NT-proBNP (pg/ml) & $6822.5 \pm 4844.9$ & $6580.3 \pm 6146.5$ & $242.3 \pm 2381.3$ \\
Paradoxical low-flow/low-gradient aortic stenosis & $3.29 \pm 0.49$ & $2.29 \pm 4.9$ & 0.004 \\
NYHA class & $63.69 \pm 8.40$ & $65.77 \pm 9.68$ & $-1.00 \pm 0.82$ \\
Left-ventricular ejection fraction (\%) & $2056.6 \pm 2474.7$ & $2253.0 \pm 2033.0$ & $-196.3 \pm 1086.9$ \\
NT-proBNP (pg/ml) & & & 0.852 \\
\hline
\end{tabular}

Values are reported as mean \pm standard deviation or $n, \%$

$T A V R$ transcatheter aortic valve replacement 
Table 7 Changes in strain after transcatheter aortic valve replacement in dependency of hemodynamic situation

\begin{tabular}{lcccc}
\hline & Before TAVR & After TAVR & $\begin{array}{l}\text { Change (paired } \\
\text { differences) }\end{array}$ & $p$ \\
\hline High-flow/high-gradient aortic stenosis & & & \\
Longitudinal & & & & \\
Peak strain (\%) & $-12.67 \pm 4.60$ & $-15.46 \pm 5.61$ & $-3.55 \pm 4.58$ & 0.048 \\
Peak strain rate systolic (\%/s) & $101.56 \pm 52.72$ & $105.49 \pm 24.36$ & $3.93 \pm 72.99$ & 0.876 \\
Peak displacement (mm) & $3.44 \pm 1.17$ & $5.09 \pm 1.46$ & $1.65 \pm 1.63$ & 0.016 \\
Peak velocity (mm/s) & $46.31 \pm 18.07$ & $47.33 \pm 12.87$ & $1.02 \pm 25.48$ & 0.907 \\
Low-flow/low-gradient aortic stenosis & & & & \\
Longitudinal & & & & \\
Peak strain (\%) & $-5.06 \pm 4.25$ & $-8.02 \pm 3.28$ & $-2.96 \pm 2.73$ & 0.045 \\
Peak strain rate systolic (\%/s) & $41.98 \pm 16.68$ & $50.43 \pm 15.83$ & $8.45 \pm 21.01$ & 0.480 \\
Peak displacement (mm) & $2.40 \pm 1.91$ & $2.72 \pm 1.79$ & $0.32 \pm 1.39$ & 0.601 \\
Peak velocity (mm/s) & $25.33 \pm 9.63$ & $37.13 \pm 11.64$ & $11.80 \pm 10.65$ & 0.042 \\
Paradoxical low-flow/low-gradient aortic stenosis & & & \\
Longitudinal & & & & \\
Peak strain (\%) & $-15.80 \pm 4.56$ & $-14.85 \pm 5.30$ & $0.96 \pm 4.94$ & 0.498 \\
Peak strain rate systolic (\%/s) & $81.0 \pm 28.48$ & $88.65 \pm 21.56$ & $7.65 \pm 21.55$ & 0.224 \\
Peak displacement (mm) & $3.98 \pm 1.21$ & $3.16 \pm 1.31$ & $-0.82 \pm 1.34$ & 0.046 \\
Peak velocity (mm/s) & $29.76 \pm 9.98$ & $29.47 \pm 9.58$ & $-0.29 \pm 9.45$ & 0.914 \\
\hline
\end{tabular}

Values are reported as mean \pm standard deviation or $n, \%$

$T A V R$ transcatheter aortic valve replacement

\section{Discussion}

We are able to show a strong correlation between CMRderived strain patterns and the hemodynamic situation in severe AS patients.

Patients with HF/HG AS showed only a mild reduction of longitudinal strain and preserved longitudinal velocity. In the LF/LG group, reduced strain and velocity could be observed. Patients in the PLF/LG group exhibited reduced longitudinal velocity despite preserved longitudinal strain. Global longitudinal velocity reliably could identify a 'low-flow' state, irrespective of other hemodynamic or morphologic findings. By evaluation of strain parameters, specific responses following TAVR concerning left-ventricular remodeling could be demonstrated. For this purpose, an emerging technique and dedicated post-processing software were validated.

Comprehensive cardiac imaging is very helpful to judge the complex situation in severe AS patients. A CMR study including 91 patients could demonstrate varying patterns of left-ventricular hypertrophy and remodeling, unrelated to the severity and duration of AS [35]. The findings reflect different left-ventricular compensatory mechanisms in response to aortic valve narrowing and confirms CMR as gold standard for the assessment of left-ventricular function. CMR-derived strain may strengthen the relevance of this modality in the diagnostic work-up of AS patients. We were able to show that CMR strain imaging offers the possibility to non-invasively assess the hemodynamic situation of individual patients. Each hemodynamic subgroup was characterized by software-specific values. This may facilitate diagnosis especially for patients with a PLF/LG situation. This is of special interest, since this challenging diagnosis accounts for approximately one-third of the total severe AS population $[8,12]$. Currently, a 'low-flow' state is defined as LVSVi $\leq 35 \mathrm{ml} / \mathrm{m}^{2}$ [25]. Calculation of this parameter depends on the formula used and often falls close to the $35 \mathrm{ml} / \mathrm{m}^{2}$ cutoff, which makes correct diagnosis of 'lowflow' still difficult $[27,28]$. It could be demonstrated that echocardiographic assessment of left-ventricular longitudinal function by Doppler-derived mitral annular peak velocity may provide helpful additional information in this situation [36]. Nevertheless, low reproducibility and the need for an appropriate 'acoustic window' limit the widespread use of echocardiographic techniques in clinical routine [16, 17]. FT CMR-derived assessment of global longitudinal strain and velocity with a high intra- and inter-reader reproducibility may be more beneficial in those patients.

Several studies have focused on left-ventricular remodeling and its correlation to outcome after aortic valve replacement and reported conflicting results [37, 38]. A study examining 50 patients undergoing either surgical or transcatheter aortic valve replacement showed a significant decrease in right and left ventricular volumes and muscle masses, as well as a little but significant increase in LVEF [36]. In contrast, another CMR study examining a small population including 27 patients before and after TAVR did not report 
significant changes in LVEF [38]. It is of note that most of the patients included in the first study already exhibited a reduced LVEF at the time of valve replacement (mean LVEF $52 \pm 12 \%$ ), while the population of the latter study mainly had a preserved LVEF $(61.5 \pm 14.5 \%)$. Assignment to a specific hemodynamically defined AS subgroup was not performed and could explain the observed discrepancies. In our population, a significant increase of LVEF could only be observed for the LF/LG group, whereas LVEF in the other subgroups was preserved. This recovery of left-ventricular function was paralleled by a significant increase in longitudinal strain and velocity. Hence, there was a significant impairment of longitudinal strain in $\mathrm{HF} / \mathrm{HG}$ AS patients despite normal LVEF. TAVR resulted in a significant recovery of longitudinal strain in this group as well, leading to similar values as in the control population with no persisting statistically significant difference. Therefore, subtle changes in leftventricular function can be detected by CMR strain imaging.

Findings of recent studies suggest that in patients with PLF/LG AS, a more advanced left-ventricular remodeling in comparison to other AS subgroups can be documented. It could be demonstrated that in this specific subset, a more pronounced deposition of fibrotic tissue occurs [35, 36]. This limits the potential for positive remodeling after valve replacement. In concordance with this concept, we did not observe an improvement in strain parameters or NTproBNP levels for this particular subgroup. This finding is in line with observational data suggesting greater benefit from TAVR for HF/HG patients in comparison to PLF/LG patients [39-41]. Nevertheless, even though benefit is lower for PLF/LG patients in comparison to other AS subgroups, a clinical improvement could also be observed for these particular patients. It could be assumed that-even without improvement of neither longitudinal strain nor LVEFreduction of left-ventricular afterload leads to a consecutive reduction of left-ventricular end-diastolic pressure and postcapillary pulmonary hypertension. This fact may explain the observation that PLF/LG patients also do better with aortic valve replacement than with conservative treatment [39-41]. The findings of our study, however, provide a rationale for investigating the optimal timing for an aortic valve intervention in case of a PLF/LG situation. Current guidelines recommend aortic valve replacement by the time symptoms occur or if LVEF is decreased below $50 \%$ in asymptomatic patients [2,5]. This concept has been challenged, especially with regard to the PLF/LG situation. The limited clinical benefit in addition to unchanged pathologic strain imaging results supports the idea that this AS subgroup might benefit from an intervention earlier in the course of the disease when the potential for reverse remodeling may be greater [42]. Therefore, it has been suggested to expand the diagnostic work-up of AS patients beyond evaluation of LVEF and symptoms and to perform a more detailed evaluation of left-ventricular function and remodeling. The assessment of cardiac mechanics by CMR strain imaging may facilitate the optimal timing for treatment and the prediction of recovery. Of course, the hypothesis generated from these considerations would have to be proven in prospective randomized trials, in which strain assessment by CMR could be used as beneficial surrogate.

Tissue tracking technologies for CMR that enable strain assessment on standard cine sequences have been emerging over the last years. They already could prove their usefulness in various clinical settings and are thought to provide deeper insights in cardiac (dys-) function [23]. Especially longitudinal strain is an early marker of impaired left-ventricular function. Reason for this finding is the fact that longitudinal function mainly is provided by subendocardial fibers [43]. This is of special interest in the setting of severe AS, since a subendocardial dysfunction is likely to occur in the presence of an increased left-ventricular afterload, even in early stages of the disease. We were able to support this assumption in our study and favor further assessment and evaluation of this parameter in AS patients.

\section{Limitations}

Several limitations need to be addressed. In our study, only longitudinal strain showed a strong correlation to the hemodynamic situation. Our results were in line with other studies proving the relevance of longitudinal strain in AS patients, even though most of the studies forming the evidence base use echocardiographic derived strain [44]. For radial and circumferential strain, values throughout the study population varied to a much greater extend. This finding might be explained by concomitant cardiac diseases, e.g. coronary artery disease that mainly affects regional left-ventricular function. Form and extent of coronary artery disease have a relevant impact on strain measurements [45]. However, it most probably also has an important impact on the way, the left ventricle adapts to AS, as well (e.g. patients with LF/LG mostly exhibit dilated left ventricles and poor ejection fractions due to coronary artery disease). Therefore, we believe that myocardial deformation assessment is a valid tool for the evaluation of left ventricular function in AS patients, irrespective of coronary artery disease. Eventually, critical coronary stenosis (e.g. left-main) usually was revascularized several weeks before AVR.

Though our study cohort is relatively small, we were able to provide strain values for the distinct AS subgroups. These values are modality and software specific and are not simply transferable to other settings. The comparability throughout different vendors, imaging modalities and post-processing software needs to be further evaluated and proven. 


\section{Conclusion}

Strain imaging by FT CMR strongly correlates to the hemodynamic situation in patients with severe AS and is able to predict remodeling after TAVR.

\section{Compliance with ethical standards}

Conflict of interest The authors state that there neither exists a conflict of interest nor that there is financial information to disclose.

Open Access This article is distributed under the terms of the Creative Commons Attribution 4.0 International License (http://creativecommons.org/licenses/by/4.0/), which permits unrestricted use, distribution, and reproduction in any medium, provided you give appropriate credit to the original author(s) and the source, provide a link to the Creative Commons license, and indicate if changes were made.

\section{References}

1. Coffey S, Cairns BJ, Iung B (2016) The modern epidemiology of heart valve disease. Heart 01:102

2. Vahanian A, Alfieri O, Andreotti F et al (2012) Guidelines on the management of valvular heart disease (version 2012): the Joint Task Force on the Management of Valvular Heart Disease of the European Society of Cardiology (ESC) and the European Association for Cardio-Thoracic Surgery (EACTS). Eur J Cardiothorac Surg 2012:42

3. Leon MB, Smith CR, Mack MJ et al (2016) Transcatheter or surgical aortic-valve replacement in intermediate-risk patients. N Engl J Med 04:37

4. Adams DH, Popma JJ, Reardon MJ et al (2014) Transcatheter aortic-valve replacement with a self-expanding prosthesis. N Engl J Med 05:370

5. Holmes DR Jr, Mack MJ, Kaul S et al (2012) ACCF/AATS/SCAI/ STS expert consensus document on transcatheter aortic valve replacement: developed in collabration with the American Heart Association, American Society of Echocardiography, European Association for Cardio-Thoracic Surgery, Heart Failure Society of America, Mended Hearts, Society of Cardiovascular Anesthesiologists, Society of Cardiovascular Computed Tomography, and Society for Cardiovascular Magnetic Resonance. J Thorac Cardiovasc Surg 2012:144

6. Möllmann H, Bestehorn K, Bestehorn M et al (2016) In-hospital outcome of transcatheter vs. surgical aortic valve replacement in patients with aortic valve stenosis: complete dataset of patients treated in 2013 in Germany. Clin Res Cardiol 2016:553-559

7. Czarny MJ, Resar JR (2014) Diagnosis and management of valvular aortic stenosis. Clin Med Insights Cardiol 8(Suppl 1):15

8. Tandon A, Grayburn PA (2013) Imaging of low-gradient severe aortic stenosis. JACC Cardiovasc Imaging 6(2):184-195

9. Carabello BA, Green LH, Grossman W, Cohn LH, Koster JK, Collins JJ Jr (1980) Hemodynamic determinants of prognosis of aortic valve replacement in critical aortic stenosis and advanced congestive heart failure. Circulation 62(1):42-48

10. Spann JF, Bove AA, Natarajan G, Kreulen T (1980) Ventricular performance, pump function and compensatory mechanisms in patients with aortic stenosis. Circulation 62(3):576-582

11. Awtry E, Davidoff R (2011) Low-flow/low-gradient aortic stenosis. Circulation 124(23): e739-e741
12. Dumesnil JG, Pibarot P, Carabello B (2010) Paradoxical low flow and/or low gradient severe aortic stenosis despite preserved left ventricular ejection fraction: implications for diagnosis and treatment. Eur Heart J 31(3):281-289

13. Weidemann F, Jamal F, Sutherland GR et al (2002) Myocardial function defined by strain rate and strain during alterations in inotropic states and heart rate. Am J Physiol Heart Circ Physiol 283(2):H792-H799

14. Weidemann F, Jamal F, Kowalski M et al (2002) Can strain rate and strain quantify changes in regional systolic function during dobutamine infusion, B-blockade, and atrial pacingimplications for quantitative stress echocardiography. J Am Soc Echocardiogr 15(5):416-424

15. Delgado V, Tops LF, van Bommel RJ et al (2009) Strain analysis in patients with severe aortic stenosis and preserved left ventricular ejection fraction undergoing surgical valve replacement. Eur Heart J 30(24):3037-3047

16. Thavendiranathan P, Grant AD, Negishi T, Plana JC, Popović ZB, Marwick TH (2013) Reproducibility of echocardiographic techniques for sequential assessment of left ventricular ejection fraction and volumes: application to patients undergoing cancer chemotherapy. J Am Coll Cardiol 61(1):77-84

17. Macron L, Lairez O, Nahum J et al (2011) Impact of acoustic window on accuracy of longitudinal global strain: a comparison study to cardiac magnetic resonance. Eur J Echocardiogr 12(5):394-399

18. Hendel RC, Patel MR, Kramer CMACCF et al (2006) ACR, SCCT, SCMR, ASNC, NASCI, SCAI, SIR, 2006 appropriateness criteria for cardiac computed tomography and cardiac magnetic resonance imaging: a report of the American College of Cardiology Foundation Quality Strategic Directions Committee Appropriateness Criteria Working Group, American College of Radiology, Society of Cardiovascular Computed Tomography, Society for Cardiovascular Magnetic Resonance, American Society of Nuclear Cardiology, North American Society for Cardiac Imaging, Society for Cardiovascular Angiography and Interventions, and Society of Interventional Radiology. J Am Coll Cardiol 2006:48

19. Bernhardt P, Rodewald C, Seeger J et al (2016) Non-contrastenhanced magnetic resonance angiography is equal to contrastenhanced multislice computed tomography for correct aortic sizing before transcatheter aortic valve implantation. Clin Res Cardiol 105(3):273-278

20. Buckert D, Kelle S, Buss S et al (2016) Left ventricular ejection fraction and presence of myocardial necrosis assessed by cardiac magnetic resonance imaging correctly risk stratify patients with stable coronary artery disease: a multi-center all-comers trial. Clin Res Cardiol (Epub ahead of print)

21. Hombach V, Merkle N, Bernhard P, Rasche V, Rottbauer W (2010) Prognostic significance of cardiac magnetic resonance imaging: Update 2010. Cardiol J 17(6):549-557

22. Wöhrle J, Merkle N, Kunze M, Cristea E, Mehran R, Rottbauer W, Stone GW (2012) Effect of bivalirudin compared with unfractionated heparin plus abciximab on infarct size and myocardial recovery after primary percutaneous coronary intervention: the horizons-AMI CMRI substudy. Catheter Cardiovasc Interv 79(7):1083-1089

23. Claus P, Omar AM, Pedrizzetti G, Sengupta PP, Nagel E (2015) Tissue tracking technology for assessing cardiac mechanics: principles, normal values, and clinical applications. JACC Cardiovasc Imaging 8(12):1444-1460

24. Andre F, Steen H, Matheis P et al (2015) Age- and gender-related normal left ventricular deformation assessed by cardiovascular magnetic resonance feature tracking. J Cardiovasc Magn Reson 17(1):25

25. Nishimura RA, Otto CM, Bonow RO et al (2014) AHA/ACC guideline for the management of patients with valvular heart disease: executive summary: a report of the American College of 
Cardiology/American Heart Association Task Force on Practice Guidelines. J Am Coll Cardiol 2014:63

26. Nishimura RA, Carabello BA (2012) Hemodynamics in the cardiac catheterization laboratory of the 21 st century. Circulation 125(17):2138-2150

27. Pibarot P, Dumesnil JG (2012) Low-flow, low-gradient aortic stenosis with normal and depressed left ventricular ejection fraction. J Am Coll Cardiol 60(19):1845-1853

28. Saikrishnan N, Kumar G, Sawaya FJ, Lerakis S, Yoganathan AP (2014) Accurate assessment of aortic stenosis: a review of diagnostic modalities and hemodynamics. Circulation 129(2):244-253

29. Kramer CM, Barkhausen J, Flamm SD, Kim RJ, Nagel E (2013) Society for Cardiovascular Magnetic Resonance Board of Trustees Task Force on Standardized Protocols. Standardized cardiovascular magnetic resonance (CMR) protocols 2013 update. J Cardiovasc Magn Reson 15(1):91

30. Schulz-Menger J, Bluemke DA, Bremerich J et al (2013) Standardized image interpretation and post processing in cardiovascular magnetic resonance: Society for Cardiovascular Magnetic Resonance (SCMR) board of trustees task force on standardized post processing. J Cardiovasc Magn Reson 15(1):35

31. Pedrizzetti G, Claus P, Kilner PJ, Nagel E (2016) Principles of cardiovascular magnetic resonance feature tracking and echocardiographic speckle tracking for informed clinical use. J Cardiovasc Magn Reson 18(1):51

32. Riffel JH, Keller MG, Aurich M et al (2015) Assessment of global longitudinal strain using standardized myocardial deformation imaging: a modality independent software approach. Clin Res Cardiol 104(7):591-602

33. Anderson RP (1994) First publications from the Society of Thoracic Surgeons National Database. Ann Thorac Surg 57(1):6-7

34. Roques F, Nashef SA, Michel P et al (1999) Risk factors and outcome in European cardiac surgery: analysis of the EuroSCORE multinational database of 19030 patients. Eur J Cardiothorac Surg 15(6):816-823

35. Dweck MR, Joshi S, Murigu T et al (2012) Left ventricular remodeling and hypertrophy in patients with aortic stenosis: insights from cardiovascular magnetic resonance. J Cardiovasc Magn Reson 14(1):50

36. Mehrotra P, Jansen K, Flynn AW, Tan TC, Elmariah S, Picard MH, Hung J (2013) Differential left ventricular remodelling and longitudinal function distinguishes low flow from normal-flow preserved ejection fraction low-gradient severe aortic stenosis. Eur Heart J 34(25):1906-1914

37. Fairbairn TA, Steadman CD, Mather AN et al (2013) Assessment of valve haemodynamics, reverse ventricular remodelling and myocardial fibrosis following transcatheter aortic valve implantation compared to surgical aortic valve replacement: a cardiovascular magnetic resonance study. Heart 99(16):1185-1191

38. La Manna A, Sanfilippo A, Capodanno D et al (2013) Left ventricular reverse remodeling after transcatheter aortic valve implantation: a cardiovascular magnetic resonance study. J Cardiovasc Magn Reson 15(1):39

39. Clavel MA, Dumesnil JG, Capoulade R, Mathieu P, Sénéchal M, Pibarot P (2012) Outcome of patients with aortic stenosis, small valve area, and low flow, low gradient despite preserved left ventricular ejection fraction. J Am Coll Cardiol 60(14):1259-1267

40. Lancellotti P, Magne J, Donal E et al (2012) Clinical outcome in asymptomatic severe aortic stenosis: insights from the new proposed aortic stenosis grading classification. J Am Coll Cardiol 59(3):235-243

41. Schewel J, Schewel D, Frerker C et al (2016) Invasive hemodynamic assessments during transcatheter aortic valve implantation: comparison of patient outcomes in higher vs. lower transvalvular gradients with respect to left ventricular ejection fraction. Clin Res Cardiol 105(1):59-71

42. Badiani S, van Zalen J, Treibel TA, Bhattacharyya S, Moon JC, Lloyd G (2016) Aortic stenosis, a left ventricular disease: insights from advanced imaging. Curr Cardiol Rep 18(8):1-9

43. Vinereanu D, Lim PO, Frenneaux MP, Fraser AG (2005) Reduced myocardial velocities of left ventricular long-axis contraction identify both systolic and diastolic heart failure-a comparison with brain natriuretic peptide. Eur J Heart Fail 7(4):512-519

44. Carasso S, Cohen O, Mutlak D et al (2011) Relation of myocardial mechanics in severe aortic stenosis to left ventricular ejection fraction and response to aortic valve replacement. Am J Cardiol 107(7):1052-1057

45. Smedsrud MK, Sarvari S, Haugaa KH et al (2012) Duration of myocardial early systolic lengthening predicts the presence of significant coronary artery disease. J Am Coll Cardiol 60(12):1086-1093 